\title{
Nonlinear Electrostatic Ion-Acoustic-Like Instabilities in a System with Two Streaming Ion Components Moving in a Background Plasma
}

\author{
L. Gomberoff*
}

\author{
Departamento de Fisica, Facultad de Ciencias, Universidad de Chile, Las Palmeras 3425, Ñuñoa, Santiago, Chile
}

\begin{abstract}
Finite amplitude Alfvén-cyclotron waves are believed to play an important role in coronal heating and nonthermal properties of velocity distribution functions. These effects are thought to be due to parametrically unstable Alfvén-cyclotron waves and electrostatic bursts of ion-acoustic like waves. It is shown here that large amplitude Alfvéncyclotron waves propagating in multi-ion plasmas with relative drift velocities between the ion-species, can lead to a new type of nonlinear electrostatic ion-acoustic like instabilities. These instabilities occur when the phase velocity of a forward propagating ion-acoustic wave supported by one ion species become equal to the phase velocity of a backward propagating ion-acoustic wave supported by another ion species. This phenomenon is only possible when relative to the background plasma there are at least two streaming ion components.
\end{abstract}

Keywords: Nonlinear waves, two-stream instability, magnetosphere.

Alfvén-cyclotron waves in plasmas with several ioncomponents have been observed in a number of space regions such as the solar wind and the Earth's magnetosphere $[1,2]$. These waves are believed to play an important role in the heating of the solar coronal plasma [3]. On the other hand, large amplitude Alfvenic fluctuations are normally associated to nonthermal features of ion velocity distribution functions [4]. It has been recently argued that the electrostatic turbulence in space plasmas consists of longitudinal waves with acoustic-like dispersion relation and it is associated with the generation of ion-beam distributions $[5,6]$.

Finite amplitude Alfvén cyclotron waves in multi-ion plasmas with relative drifts among the ion components, have been shown to lead to several important effects: (a) parametric instabilities of the decay, beat wave, and modulational type [7], (b) important changes to the linear response of the systems [8,9], and, (c) nonlinear ion-acoustic-like electrostatic instabilities $[10,11]$. These instabilities have been shown to occur when the phase velocity of forward and backward propagating ion-acoustic waves relative to the supporting ion species, become equal [10.11]. These types of instabilities can only occur when at least two of the ion species have a nonzero relative drift velocity $[10,11]$.

We shall show here that when there are more than two ion species drifting relative to each other, another type of electrostatic ion-acoustic-like instabilities can exist. These instabilities occur when the phase velocity of forward propagating ion-acoustic waves supported by one ion species overlaps with backward propagating ion acoustic waves supported by a different drifting ion species due to the action of the large amplitude Alfvén-cyclotron wave. This instability is similar to that triggered by linear ion-acoustic waves when the phase velocity of the Alfvén-ion-cyclotron wave

*Address correspondence to this author at the Departamento de Fisica, Facultad de Ciencias, Universidad de Chile, Las Palmeras 3425, Ñuñoa, Santiago, Chile; E-mail: lgombero@uchile.cl associated to one ion species overlaps the phase velocity of the ion-cyclotron waves associated to another species drifting relative to the former [7, 12-15]. However, the physical features of both types of instabilities are completely different. There are also classical linear two-stream instabilities where the overlapping between ion acoustic waves is between ion acoustic waves supported by different drifting ionspecies [16]. However, the nonlinear ion-acoustic-like instabilities require the presence of large amplitude Alfvén waves and at least three ion components. Moreover, the instability frequency and wavenumber range are different, and they occur for different values of the parameters.

A nonlinear instability of the kind discussed here but in a completely different parameter space region, can also occur in one beam plasma. This instability will be discussed somewhere else.

In order to illustrate the results, we shall use a magnetospheric plasma model consisting of electrons, cold protons (pc), drifting hot protons (hp), $\mathrm{He}^{+}$and $\mathrm{O}^{+}$ions. We shall use fluid theory throughout, although it has been recently shown that kinetic effects cannot be neglected even for low $\beta_{1}=4 \pi n_{p} K_{1} / B_{0}^{2}$ plasmas [6] ( $n_{p c}$ is the proton core density, $T_{1}$ the temperature of the 1th. ion-species, and $\mathrm{B}_{0}$ the external magnetic field). However, for low enough $\beta$-values, fluid theory should provide a satisfactory description of the plasma. A system like the one used here has been considered before [15] in order to show that parametric decays of finite amplitude Afvén-cyclotron waves are drastically affected by the drift velocities of the ion species. It was also shown there that electrostatic nonlinear ion-acoustic like instabilities between ion-acoustic waves supported by the same ion species can also exist in such systems. In contrast, it is shown here that nonlinear ion-acoustic like instabilities can also exist between ionacoustic waves supported by different ion species.

The nonlinear dispersion relation for drifting hot protons, $\mathrm{He}^{+}$, and $\mathrm{O}^{+}$ions relative to the cold core protons, can be written in the following form $[14,15]$, 


$$
L_{+} L_{-} Q+L_{+} T_{-}^{-}-L_{-} T_{+}^{+}+\left(T_{+}^{-} T_{-}^{+}-T_{+}^{+} T_{-}^{-}\right) / Q=0
$$

The definitions of the various terms are,

$$
\begin{aligned}
& T_{ \pm}^{ \pm}=\frac{\alpha_{p c}^{ \pm}}{A_{p c}}\left(R_{ \pm p c} Q+P_{\mp}\right)+\frac{\alpha_{h p}^{ \pm}}{A_{h p}}\left(R_{ \pm h p} Q+\eta_{h p} P_{ \pm}\right)+ \\
& \frac{\alpha_{H e^{+}}^{ \pm}}{A_{H e^{+}}}\left(R_{ \pm H e^{+}} Q+\eta_{H e^{+}} P_{ \pm}+\frac{\alpha_{O^{+}}^{ \pm}}{A_{O^{+}}}\left(R_{ \pm O^{+}} Q+\eta_{O^{+}} P_{\mp}\right),\right. \\
& L_{ \pm}=y_{ \pm}^{2}-\frac{x_{ \pm p c}^{2}}{\psi_{ \pm}}-\frac{\eta_{h p} x_{h p}^{2}}{\psi_{ \pm h p}}-\frac{4 \eta_{H e^{+}}}{\psi_{ \pm H e^{+}}}-\frac{16 \eta_{O^{+}} x_{O^{+}}^{2}}{\psi_{O^{+}}},
\end{aligned}
$$

where

$$
\begin{aligned}
& R_{ \pm l}=\eta_{l} \frac{m_{l}}{m_{p}} \frac{y_{ \pm} x_{l}}{2 \psi_{0 l}}\left(x_{0 l}-\frac{y x_{0 l}^{2}}{y_{0} x_{l}}+\frac{x_{ \pm l}}{\psi_{ \pm l}}\right), \\
& Q=1-\beta_{e} y^{2}\left(\frac{1}{A_{p c}}+\frac{\eta_{h p}}{A_{h p}}+\frac{\eta_{h p}}{A_{h p}}+\frac{\eta_{h p}}{A_{h p}}\right), \\
& P_{ \pm}=\beta_{e} y^{2}\left(\frac{R_{ \pm p c}}{A_{p c}}+\frac{R_{ \pm h p}}{A_{h p}}+\frac{R_{ \pm H e^{+}}}{4 A_{H e^{+}}}+\frac{R_{ \pm O^{+}}}{16 A_{O^{+}}}\right), \\
& A_{l}=\left[x_{l}^{2}-\left(\frac{\beta_{l} m_{p}}{m_{l}}\right) y^{2}\right]+\frac{B^{2}}{B_{0}^{2}} \frac{x_{l}^{2}}{\psi_{0 l} \psi_{-l} \psi_{-l}}, \\
& \alpha_{l}^{ \pm}=\frac{B^{2}}{B_{0}^{2}} \frac{x_{0 l}^{2} y_{ \pm} \psi_{ \pm l}-x_{ \pm l}^{2} y_{o l} \psi_{0 l}}{y_{0} y_{ \pm} \psi_{0 l} \psi_{ \pm l}},
\end{aligned}
$$

and

$$
\begin{aligned}
& \beta_{l}=\frac{4 \pi n_{p c} \gamma_{l} K T_{l}}{B_{0}^{2}}, \\
& \beta_{e}=\frac{4 \pi n_{p c} \gamma_{l} K T_{e}}{B_{0}^{2}}, \\
& x={ }^{\omega_{A}} / \Omega_{p}, \\
& y={ }^{k v_{A}} / \Omega_{p}, \\
& x_{ \pm}=x_{0} \pm x, \\
& y_{ \pm}=y_{0} \pm y, \\
& x_{ \pm h p}=x_{ \pm}-y_{ \pm} U_{h p,}, \\
& x_{ \pm H e^{+}}=x_{ \pm}-y_{ \pm} U H e^{+}, \\
& x_{p h^{+}}=x-y U_{p h}, \\
& x_{H e^{+}}=x-y U_{H e^{+}}, \\
& x_{O^{+}}=x-y U_{O^{+}}, \\
& \psi_{o l}=1-\frac{m_{l}}{m_{p}} x_{0}, \\
& \psi_{ \pm l}=1-\frac{m_{l}}{m_{p}} x_{ \pm l} .
\end{aligned}
$$

In these definitions $\mathrm{x}_{0}$ and $\mathrm{y}_{0}$ are the frequency and wavenumber of the finite amplitude wave normalized like $\mathrm{x}$ and $y, U_{1}=v_{1} / v_{A}$, where $v_{1}$ is the drifting velocity of species $\left\{\right.$ it 1 \} relative to the cold protons, $\mathrm{v}_{\mathrm{A}}=\mathrm{B}_{0} /\left(4 \pi \mathrm{n}_{\mathrm{pc}} \mathrm{m}_{\mathrm{p}}\right)^{1 / 2}$, and $\eta_{1}=n_{1} / n_{p c}$ for $\mathrm{l}=h p, \mathrm{He}^{+}, \mathrm{O}^{+}$.

Except for the first term in (1), all other terms vanish for zero pump wave intensity, $A=\left(B / B_{0}\right)^{2}$, where $B$ is the magnetic field of the finite amplitude wave. Hence, for zero pump wave intensity, the dispersion relation reduces to,

$L_{+} L_{-} Q=0$.

From the last equation it follows that for $A=0$, the pump wave satisfies $\mathrm{L}_{ \pm}=0$,

and

$Q=0$.

The last equation gives the ion-acoustic sound waves present in the system. Since the electrons and cold proton, are much more dense than the hot protons, the $\mathrm{He}^{+}$and $\mathrm{O}^{+}$ ions, the solutions of (4) are approximately given by:

$$
\begin{aligned}
& x \cong \pm\left(\beta_{e}+\beta_{p c}\right)^{1 / 2} y, \\
& x-y U_{h p} \cong \pm\left(\beta_{h p}\right)^{1 / 2} y, \\
& x-y U_{H e_{+}} \cong \pm\left(\beta_{H e^{+}}\right)^{1 / 2} y, \\
& x-y U_{O^{+}} \cong \pm\left(\beta_{O^{+}}\right)^{1 / 2}
\end{aligned}
$$

Thus, there are six ion-acoustic modes in the system. The first two, given by (4), are ordinary sound waves supported by the cold protons. The second two, given by (5), are supported essentially by the hot protons, and those given by (6) and (7) are supported mainly by the $\mathrm{He}^{+}$and $\mathrm{O}^{+}$ions.

We shall now solve (1) numerically for a magnetospheric-like plasma consisting of $\mathrm{n}_{\mathrm{e}}=12.4 \mathrm{~cm}^{-3}, \mathrm{n}_{\mathrm{pc}}=10 \mathrm{~cm}^{-3}$, $\mathrm{n}_{\mathrm{hp}}=1.1 \quad \mathrm{~cm}^{-3}, \quad \mathrm{n}_{\mathrm{He}+} 1.2 \quad \mathrm{~cm}^{-3}, \quad \mathrm{n}_{\mathrm{O}+}=0.1 \quad \mathrm{~cm}^{-3}, \quad \mathrm{KT}_{\mathrm{pc}} / 2$ $=\mathrm{KT}_{\mathrm{He}+} / 2=\mathrm{KT}_{\mathrm{O}+}=5 \mathrm{eV}, \mathrm{KT}_{\mathrm{hp}} / 2=17 \mathrm{keV}, \mathrm{B}_{0}=130 \mathrm{nT}$. These values are consistent with the geostationary region explored by GEOS 1 and 2 (see, e. g. [17]).

In Fig. (1) we have plotted the nonlinear dispersion relation (1), $\mathrm{x} v s \mathrm{y}$, for $\mathrm{x}_{0}=0.05$ with corresponding $\mathrm{y}_{0}=065, \mathrm{~A}=0$, $\mathrm{U}_{\mathrm{O}}{ }^{+}=0.3, \mathrm{U}_{\mathrm{He}+}=0.12$, and $\mathrm{U}_{\mathrm{hp}}=0$. The other parameters are $\eta_{\mathrm{hp}}=0.11, \eta_{\mathrm{He}+}=0.12, \eta_{\mathrm{O}+}=0.01, \beta_{\mathrm{He}+}=\beta_{\mathrm{O}+}=0.0033, \beta_{\mathrm{hp}}=11.36$ and $\beta_{\mathrm{e}}=0.0011 \$$. The nonlinear dispersion relation has 16 roots. However, since we are interested only on electrostatic instabilities, we shall refer mainly to them. Thus, in the figure we have denoted the solutions of (11) by \pm pcs. These are ion-acoustic waves supported by the background plasma and are moving forward and backward relative to the background along the external magnetic field. Likewise, we have denoted by \pm Hes the solutions of (12) which correspond to ionacoustic waves moving forward and backward relative to the $\mathrm{He}^{+}$ions drifting in the direction of the external magnetic field. These waves are supported by the $\mathrm{He}^{+}$ions. Finally, the solutions of 13 are denoted by \pm Os. These are ionacoustic waves supported by the $0^{+}$ions moving forward and backward relative to these ions in the direction of the drift velocity. The square of the amplitude of the finite amplitude 
wave is $A=\left(B / B_{0}\right)^{2}$, where $B$ is the finite Alfvén wave magnetic field, and $\mathrm{B}_{0}$ is the external magnetic field. The other roots correspond to upper and lower sideband waves. We have labeled by $-\mathrm{O}$ only one of them, corresponding to $-\mathrm{O}^{+}$ and belonging to a lower sideband wave, $\mathrm{x}_{-}=\mathrm{x}_{0^{-}} \mathrm{x}$, in order to illustrate one of these roots.

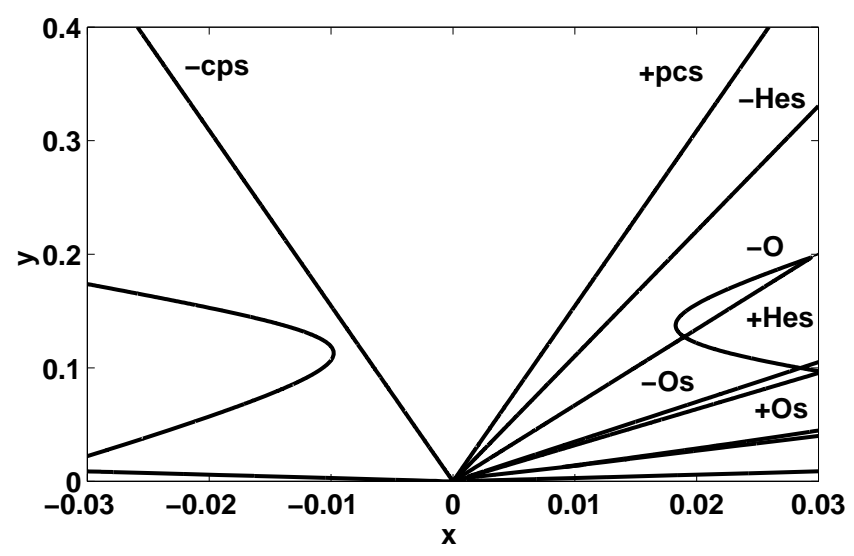

Fig. (1).

In Fig. (2) we have raised the pump wave amplitude to $\mathrm{A}=0.009$ showing that $+\mathrm{pcs}$ and $-\mathrm{Hes}$ approach each other in the region close to the origin, shown by the arrow. As A increases, these two roots overlap giving rise to the nonlinear ion-acoustic like waves. This situation is illustrated in Fig. (3) for $\mathrm{A}=0.02$, where the gap between the point $\mathrm{b}$ and the origin shows that these two roots, (+pcs, -Hes), become complex conjugate in this region giving rise to the instability. For this particular A-value, the maximum growth rate of the instability is $\gamma_{\mathrm{m}}=\operatorname{Im}(\mathrm{x})_{\mathrm{m}} / \Omega_{\mathrm{p}}=1.8 \times 10^{-3}$. As A continues $\mathrm{t}$ $\mathrm{o}$ increase, the maximum growth rate increases too. Thus, for example, for $A=0.5$, the maximum growth rate is $\gamma_{\mathrm{m}}=1.8 \mathrm{x}$ $10^{-3}$. For $0.08 \leq \mathrm{A} \leq 1$, all nonlinear instabilities are of the same order, and therefore, the instability discussed here is as important as the others. The other instabilities in this region are an ion-acoustic like one between (-pcs, +pcs), and the decay instabilty between $(-\mathrm{O},+\mathrm{Os})[15]$, as shown in Fig. (4) for $\mathrm{A}=0.065$.

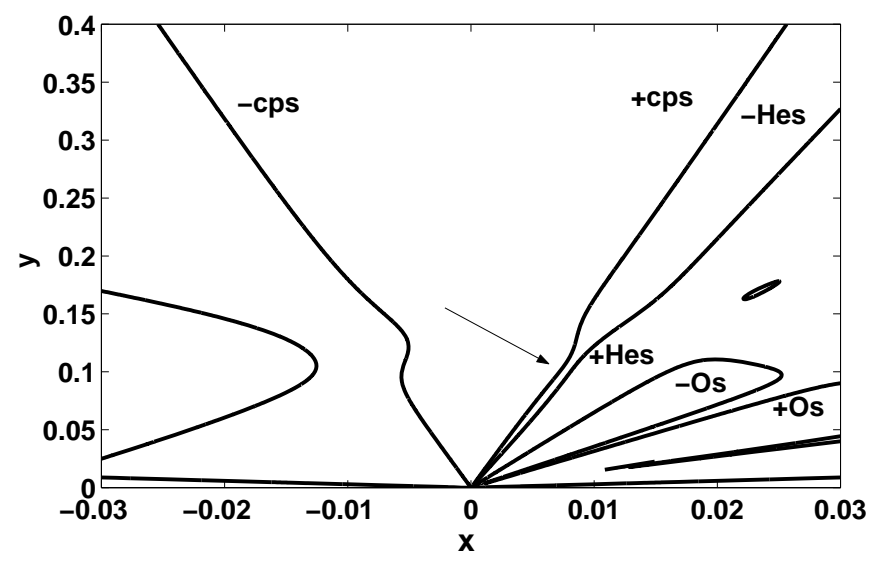

Fig. (2).

In the example of the above figures, the same pattern occurs until the -Hes root touches the root denoted by $-\mathrm{O}$ in Fig. (1). For other values of $\mathrm{U}_{\mathrm{O}}{ }^{+}$, the corresponding $\mathrm{U}_{\mathrm{He}}{ }^{+}-$ values are different, but the same pattern is found. On the other hand, $\mathrm{U}_{\mathrm{He}}{ }^{+}$can be as small as $\mathrm{U}_{\mathrm{He}}{ }^{+} \cong 0.02$. The only condition is that + Hes should be the the right of + cps. When $+c p s$ is to the right of $+\mathrm{Hes}$, the nonlinear ion-acoustic like instability occurs between $-\mathrm{Hes}$ and $+\mathrm{Hes}$, namely, between ion-acoustic waves supported by the same ion-species $[10$, 11]. In general, this instability requires the drift velocity of one of the drifting ion-species be much larger than the drift velocity of the other drifting species.

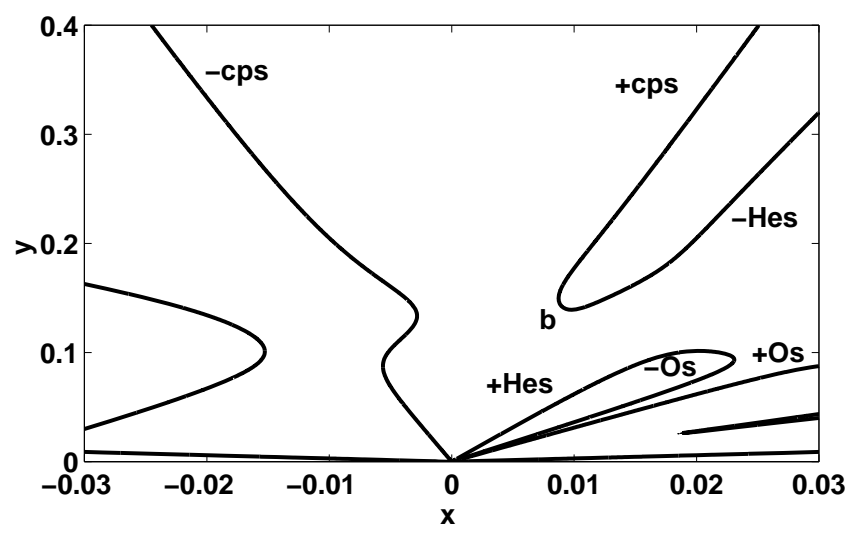

Fig. (3).

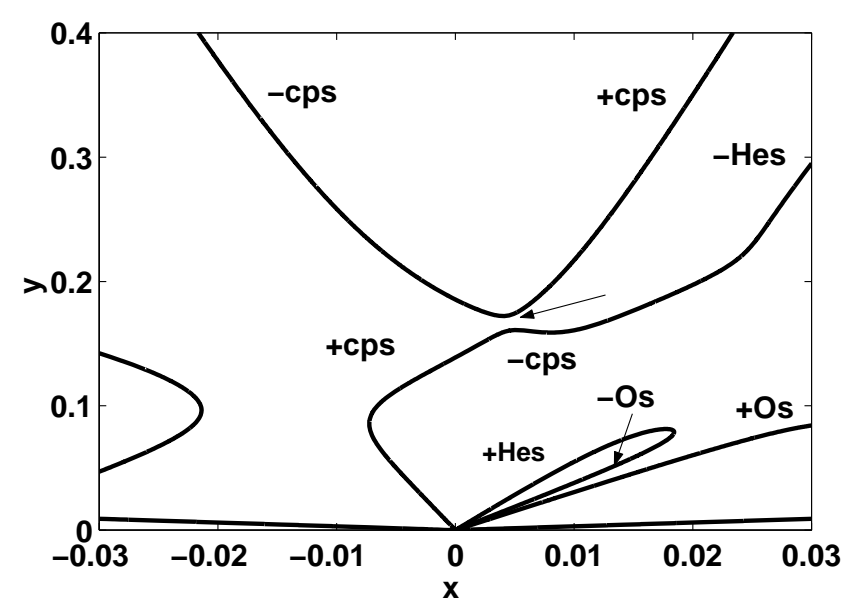

Fig. (4).

The nonlinear instability described here is similar to the linear instability discussed in [7,13], in the sense that they occur when the ion-acoustic waves supported by one species overlap with the phase velocity of those supported by another species (see also [16]). However, their properties are completely different. In the former case the waves are linear and the instability range extends from $\omega=0$ up to infinity. The latter, however, is nonlinear, $\mathrm{A} \neq 0$, and their frequency range depends on the ampli-tude of the finite amplitude wave. Moreover, these waves require at least two ion-, drifting relative to a third one. The ion species need not be different but, of course, they must have different drift velocities among themselves.

By using a series of $1 \mathrm{D}$ hybrid simulation codes, it has been recently argued that kinetic effects cannot be ignored in the study of parametric instabilities [6]. Our treat-ment, however, is based only on fluid theory. Nevertheless, we believe that for the low beta-values of the magnetospheric example used in this letter, kinetic effects can be safely ignored. The beta-values used in [6] are of the order of 0.02 , while in our case they are of the order of $10^{-3}$. The presence 
of a hot proton component with a large $\beta=11.36$ is probably strongly Landau damped. Moreover, the dispersion branches associated to this ion-component can be shown not to participate in the effect considered here, as it also follows from the figures. In fact, one can neglect the hot proton component altogether and the effect remains unchanged. However, a kinetic study of these effects is necessary, and kinetic hybrid simulations should be helpful.

As pointed out above, by using numerical simulations involving hybrid codes, it has been shown recently that the electrostatic turbulence in space plasmas consists of longitudinal waves with acoustic-like dispersion relation and it is associated with the generation of ion-beam plasma distributions, providing, thus, a novel explanation for the electrostatic bursts of ion-acoustic activity [5]. Therefore, electrostatic nonlinear ion-acoustic-like instabilities can play a major role in space plasmas.

\section{REFERENCES}

[1] Roberts WA, Goldstein ML, Klein LW. The amplitude of interplanetary fluctuations: stream structure, heliocentric distance, and frequency dependence. J Geophys Res 1990; 95: 4203-16.

[2] Anderson BJ, Erlandson RE, Zanetti LJ. A statistical study of Pc12 magnetic pulsations in the equatorial magnetosphere, 1, Equatorial occurrence distributions. J Geophys Res 1992: 97: 3075-88.

[3] Handson B, Voss D. Sunrise. Science 2007; 318:1571, DOI: 10.1126/science.318.5856.1571.

[4] Marsch E. Kinetic physics of the solar corona and solar wind. Living Rev Solar Phys 2006; 3: 1-100.

[5] Valentini F, Veltri P, Califano F, et al. Cross-scale effects in solarwind turbulence. Phys Rev Lett 2008; 101(2): 025006 1-4.
[6]

Araneda JA, Marsch E, Viñas AF. Proton core heating and beam formation via parametrically unstable Alfven-cyclotron waves. Phys Rev Lett 2008; 100(12): 125003 1-4.

[7] Hollweg JV, Esser R, Jayanti V. Modulational and decay instabilities of Alfvén waves: Effect of streaming $\mathrm{He}^{+}$ions. J Geophys Res 1993; 98: 3491-500.

[8] Gomberoff L. Stabilization of linear ion beam right-hand polarized instabilities by nonlinear Alfvén/ion-cyclotron waves. J Geophys Res 2003; 108: A6, doi:10.1029/2003JA009837.

[9] Araneda JA, Gomberoff L. Stabilization of right-hand polarized beam plasma instabilities due to a large-amplitude left-hand polarized wave: A simulation study. J Geophys Res 2004; 109: 01106, doi:10.1029/2003JA010189.

[10] Gomberoff L, HoyosJ, Brinca A, et al. Electrostatic instabilities induced by large-amplitude left-hand polarized waves. J Geophys Res 2004; 109: A07108 doi:10.1029/2004JA010466.

[11] Gomberoff L, Hoyos J, Brinca AL. Behaviour of linear beamplasma instabilities in the presence of finite amplitude circularly polarized waves. Braz J Phys 2004; 34: 547-55.

[12] Hoyos J, Gomberoff L. Influence of nonlinear circularly polarized wave on linear electromagnetic and electrostatic beam-plasma waves. Astrophys J 2005; 630: 11125-32.

[13] McKenzie JF, Marsch E, Sauer L, et al. Wave and stability properties in multi- ion species with applications to solar wind and flows. Ann Greophys. 1993; 11: 341-48.

[14] Gomberoff L. Linear and nonlinear electromagnetic and electrostatic instabilities in a plasma with two ion beams. J Geophys Res 2008; 113: A02102, doi:10.1029/2007JA012723.

[15] Gomberoff L. Electrostatic instabilities triggered by finite amplitude Alfvén- cyclotron waves and relative drifts among the ion components in the magnetosphere. J Geophys Res 2008; 113: A10207 doi:10,1029/2008JA013378.

[16] Muschieti L, Roth I. Ion two-stream instabilities in the auroral cceleration region. J Geophys Res 2008; 113: A08201, doi:10. 1029/2007/JA013005.

[17] Young DT, Perraut S, Roux A, et al. Wave-particle interactions near $\Omega_{\mathrm{He}}{ }^{+}$observed on GEOS 1 and 2. 1 Propagation of ion cyclotron waves in $\mathrm{He}^{+}$-rich plasma. J Geophys Res 1981; 86: 6755-72. 